\title{
Abnormal Cerebellar Volume in Patients with Remitted Major Depression with Persistent Cognitive Deficits
}

\author{
Malte S. Depping ${ }^{1} \cdot$ Mike M. Schmitgen ${ }^{1} \cdot$ Claudia Bach $^{1} \cdot$ Lena Listunova $^{1} \cdot$ Johanna Kienzle $^{1} \cdot$ Katharina M. Kubera $^{1}$. \\ Daniela Roesch-Ely ${ }^{1} \cdot$ R. Christian Wolf ${ }^{1}$ (D)
}

Published online: 8 July 2020

(C) The Authors 2020, corrected publication 2021

\begin{abstract}
Cerebellar involvement in major depressive disorder (MDD) has been demonstrated by a growing number of studies, but it is unknown whether cognitive functioning in depressed individuals is related to cerebellar gray matter volume (GMV) abnormalities. Impaired attention and executive dysfunction are characteristic cognitive deficits in MDD, and critically, they often persist despite remission of mood symptoms. In this study, we investigated cerebellar GMV in patients with remitted MDD (rMDD) that showed persistent cognitive impairment. We applied cerebellum-optimized voxel-based morphometry in 37 patients with rMDD and with cognitive deficits, in 12 patients with rMDD and without cognitive deficits, and in 36 healthy controls (HC). Compared with HC, rMDD patients with cognitive deficits had lower GMV in left area VIIA, crus II, and in vermal area VIIB. In patients with rMDD, regression analyses demonstrated significant associations between GMV reductions in both regions and impaired attention and executive dysfunction. Compared with HC, patients without cognitive deficits showed increased GMV in bilateral area VIIIB. This study supports cerebellar contributions to the cognitive dimension of MDD. The data also point towards cerebellar area VII as a potential target for non-invasive brain stimulation to treat cognitive deficits related to MDD.
\end{abstract}

Keywords Magnetic resonance imaging $\cdot$ Major depressive disorder $\cdot$ Cerebellum $\cdot$ Cerebellar gray matter $\cdot$ Attention $\cdot$ Executive function

\section{Introduction}

Cognitive dysfunction is a highly relevant symptom domain in major depressive disorder (MDD), affecting about twothirds of acutely depressed patients [1]. In patients with MDD, cognitive impairment independently mediates functional outcomes, e.g., workforce performance [2]. Cognitive dysfunction in depression typically includes deficits of attention and executive functions $[1,3]$. Importantly, in 30-50\% of patients with MDD, cognitive dysfunction persists despite remission of mood symptoms $[1,4]$. If persistent in remitted

Electronic supplementary material The online version of this article (https://doi.org/10.1007/s12311-020-01157-z) contains supplementary material, which is available to authorized users.

R. Christian Wolf

christian.wolf@med.uni-heidelberg.de

1 Center for Psychosocial Medicine, Department of General Psychiatry, University of Heidelberg, Vossstr. 4, 69115 Heidelberg, Germany individuals with MDD, such impairments may mediate an increased risk of major depression relapse [5-7]. Cognitive dysfunction in MDD is difficult to treat with little evidence for procognitive effects of antidepressant medication [8]. Non-pharmacological interventions, such as cognitive remediation therapy, may be effective, yet data in this regard is scarce and treatment benefits are poorly defined [9].

Against this background, there is an urgent need for a more detailed understanding of the neural mechanisms of cognitive dysfunction in MDD. Using structural and functional magnetic resonance imaging (MRI), neural correlates of cognitive dysfunction in depression have been identified by numerous studies, including meta-analyses [10-13]. Although the past decade has witnessed an increased interest in cerebellar involvement in depression, potential cerebellar contributions to cognitive dysfunction in MDD have rarely been investigated [14]. Since approximately half of the cerebellar cortex is associated with non-motor functions, i.e., cognitive, affective, and selfreferential functions [15], this may be a serious omission.

Functional MRI (fMRI) analyses of cerebro-cerebellar connectivity in large human samples have revealed a clear 
cerebellar topography, i.e., parallel neural circuits that the cerebellar subregions form with different functional areas of the cerebral cortex and with different subcortical structures [15]. There is a special role for cerebellar area VII. Unlike all other cerebellar areas, area VII is not connected with the somatomotor system but exclusively communicates with cerebral structures that support cognitive, affective, and selfreferential processes [16]. Additional non-motor representations are located in cerebellar areas VI and IX [16].

Investigations of cerebellar structure and function with MRI require optimized data analysis methods. Image registration should employ cerebellum-optimized templates, since the use of conventional templates results in anatomically imprecise findings [17, 18]. A small number of cerebellumoptimized MRI investigations in MDD have been published so far. Voxel-based morphometry (VBM) with cerebellumoptimized data analysis has been applied by two studies [19, 20]. Cerebellar resting perfusion has been examined by one fMRI study [21]. In patients with acute depression, abnormal gray matter volume (GMV) has been demonstrated in area IX, a region belonging to the so-called default-mode network (DMN) [20]. Additionally, in patients with acute MDD, abnormal resting perfusion within area VIIA has been associated with depressive symptom load [21]. However, neither of those studies specifically addressed cognitive functions. A few fMRI studies have investigated cerebellar intrinsic connectivity, yet cerebellum-optimized data analysis was not applied in any of those studies $[14,22-26]$. Keeping this important limitation in mind, there is preliminary evidence for abnormal resting-state connectivity of area VIIA with cerebral components of the so-called cognitive control network $(\mathrm{CCN})$ in patients with acute depression $[14,24,25]$.

This study investigated structural cerebellar correlates of persistent cognitive dysfunction in patients with remitted depression. Cognitive functioning in mental disorders can be conceptualized as a dimensional construct [27]. Along a spectrum of functioning, this study included depressed patients with a more severe impairment of attention and executive functions. The study aimed at cerebellar mechanisms that contribute to the cognitive dimension of MDD. The study subjects were participants in a project that evaluated cognitive remediation therapy in remitted MDD [28]. For this study, patients were investigated at baseline, i.e., prior to cognitive training. Based on evidence for cognitive processes predominantly subserved by area VII, and based on cumulative data suggesting abnormal structure and function of this cerebellar subregion in acute depression, we hypothesized that patients with remitted depression and persistent cognitive deficits would show abnormal gray matter volume (GMV) of area VII. We also expected that area VII GMV in patients would be significantly associated with cognitive performance, as measured by a comprehensive neuropsychological test battery.

\section{Material and Methods}

\section{Patient Characteristics}

This study was part of a project that examined the effects of cognitive training in patients with remitted MDD. Patient recruitment and selection have been described in detail elsewhere, as have been the preliminary behavioral outcomes of that project [28]. Briefly, patients were diagnosed at the Department of General Psychiatry, University Hospital Heidelberg, using the Structured Clinical Interview for DSM-IV (SCID-I). Patients with a history of MDD and remission at the time of the examination were included in the study. Four neuropsychological domains were evaluated: attention, processing speed, executive functions, and learning/memory, for details see section "Neuropsychological Evaluation." Patients were considered to be cognitively impaired, if they showed deficits (standardized test values of $z<-1$ ) in at least two out of six cognitive subdomains (alertness, selective attention, divided attention, working memory, inhibition, planning; for details see section "Neuropsychological Evaluation"). Only subjects between 18 and 60 years of age and with an IQ $>80$ according to the Multiple Choice Vocabulary Test (MWT-B, [29]) were included. Exclusion criteria were comorbid psychiatric disorders according to DSM-IV (except dysthymia), neurological disease, or past traumatic brain injury. MRI examinations were performed prior to cognitive remediation training. MRI examinations were carried out in 37 patients with remitted MDD and persistent cognitive dysfunction (rMDD with c.d.), in 12 patients with remitted MDD and without cognitive deficits (rMDD without c.d.), and in 36 healthy volunteers (HC), see Table 1. The median estimated duration of current remissionaccording to the patient's assessment—was 7.6 months in patients with persistent cognitive deficits and 8.6 months in patients without cognitive deficits $(p=0.56)$. At the time of study enrollment, 47 patients were treated as outpatients, and 2 patients were treated at a psychiatric day hospital. Any psychotropic medication established in patients' regular treatment remained unchanged. Of all patients with persistent cognitive deficits, 20 received psychotropic medication (venlafaxine $=5$, escitalopram $=3$, citalopram $=3$, vortioxetine $=2$, agomelatine $=1$, amitriptyline $=1$, bupropion $=1$, duloxetine $=$ 1 , fluvoxamine $=1$, quetiapine $=1$, sertraline $=1$ ). Of all patients without cognitive deficits, 5 received psychotropic medication (sertraline $=2$, citalopram $=1$, fluoxetine $=1$, venlafaxine $=1$ ). Of all patients receiving psychotropic medication, all but two patients were on a monotherapy. No patient had ever received electroconvulsive therapy. All control subjects were medication-free (except for contraceptives). All subjects were right-handed. The study was approved by the local ethics committee (Heidelberg University) and carried out in accordance with the Helsinki Declaration. Written informed consent was obtained from all participants. 
Table 1 Demographics and clinical variables for patients with remitted major depression (rMDD) with or without persistent cognitive deficits (c.d.), as well as for healthy controls (HC). HAMD, Hamilton Depression Rating Scale; $s d$, standard deviation; n.a., not applicable

\begin{tabular}{|c|c|c|c|c|c|c|c|}
\hline & \multicolumn{2}{|c|}{$\begin{array}{l}\text { rMDD with c.d. } \\
(n=37)\end{array}$} & \multicolumn{2}{|c|}{$\begin{array}{l}\text { rMDD without c.d. } \\
(n=12)\end{array}$} & \multicolumn{2}{|c|}{$\mathrm{HC}(n=36)$} & \multirow[b]{2}{*}{$p$ value } \\
\hline & Mean & sd & Mean & sd & Mean & sd & \\
\hline Age (years) & 45.0 & 12.7 & 40.6 & 12.8 & 40.5 & 12.2 & $0.276^{\mathrm{a}}$ \\
\hline Gender (m/f) & $15 / 22$ & n.a. & $1 / 11$ & n.a. & $12 / 24$ & n.a. & $0.121^{\mathrm{b}}$ \\
\hline Education (years) & 11.9 & 1.7 & 12.3 & 0.9 & 11.7 & 1.5 & $0.464^{\mathrm{a}}$ \\
\hline Duration of illness (years) & 14.5 & 12.9 & 15.6 & 11.9 & n.a. & n.a. & $0.803^{\mathrm{c}}$ \\
\hline Number of episodes & 4.2 & 7.1 & 3.5 & 3.2 & n.a. & n.a. & $0.752^{\mathrm{c}}$ \\
\hline HAMD & 4.9 & 3.6 & 5.8 & 4.1 & 0.4 & 0.8 & $<0.001^{\mathrm{a}}$ \\
\hline
\end{tabular}

${ }^{\mathrm{a}}$ ANOVA

${ }^{\mathrm{b}}$ Chi-square test

${ }^{c} t$ test

\section{Neuropsychological Evaluation}

Four cognitive domains were tested: (1) Attention was assessed using the Perception and Attention Functions Battery (WAF). Three different attention domains were tested, i.e., alertness (WAFA, performance measure [PM]: response time), selective attention (WAFS, PM: number of errors), and divided attention (WAFG, PM: number of omissions). (2) Processing speed was assessed using the Trail Making Test-A (TMT-A, PM: completion time) and the Digit Symbol Substitution Test (DSST, PM: number of correct assignments). (3) Assessment of executive functions included tests to evaluate working memory (N-Back verbal, PM: number of errors), inhibition (Go-Nogo, PM: response time, number of errors), cognitive flexibility (Trail Making Test-B, PM: completion time), and planning (Tower of London (ToL), PM: number of correctly solved tasks). (4) Assessment of learning and memory included tests for verbal memory (California Verbal Learning Test (CVLT), PM: number of words recalled, immediate and delayed recall accuracy) and figural memory (Figural Memory Test, PM: number of figures recalled, immediate and delayed recall accuracy). The neuropsychological tests were included in the computerized "Vienna Test System" (Schuhfried GmbH, 2012, http://www. schuhfried.at). Raw test values were $z$-transformed and polarized into one direction with higher $z$-values indicating better cognitive performance. Individual test scores were averaged to form four domain scores (attention, processing speed, executive functions, learning, and memory), see Table 2. Domain scores were additionally averaged to form a general cognitive composite score, see Table 2.

\section{MRI Data Acquisition}

MRI scans were acquired using a 3 Tesla Siemens Magnetom TIM Trio scanner, located at the Department of Neuroradiology, University Hospital Heidelberg. T1-
MPRAGE sequences were acquired with the following parameters: $\mathrm{TE}=2.52 \mathrm{~ms} ; \mathrm{TR}=1900 \mathrm{~ms}$; $\mathrm{TI}=900 \mathrm{~ms}$; FOV $=256 \mathrm{~mm}$; slice thickness $=1 \mathrm{~mm}$; resolution $=1.0 \times$ $1.0 \times 1.0 \mathrm{~mm}$; number of slices $=256$.

\section{MRI Data Analysis}

The Statistical Parametric Mapping Software, Version 12 (SPM 12, http://www.fil.ion.ucl.ac.uk/spm), implemented in MATLAB R2019a, was used to analyze MRI data. The Spatially Unbiased Infratentorial Toolbox (SUIT, http:// www.diedrichsenlab.org/ima-ging/suit.htm) was used for cerebellum-optimized VBM. Individual T1-weighted sequences were controlled for scanner artifacts and the image origin was set at the anterior commissure. The infratentorial structures, i.e., cerebellum and brain stem, were isolated from the surrounding tissue. Using the unified segmentation approach [30], the infratentorial structures were segmented into gray matter (GM), white matter, and cerebrospinal fluid. Using the Diffeomorphic Anatomical Registration Through Exponentiated Lie Algebra (DARTEL) algorithm [31], the individual GM segments were registered onto the SUIT atlas template. In order to preserve individual anatomical differences, the GM probability maps were modulated using the deformation fields that resulted from the registration procedure, providing GMV maps [32]. The GMV maps were smoothed with a Gaussian kernel of 4-mm full width at halfmaximum (FWHM) [33].

Voxel-wise statistical analyses were applied to assess the effect of cognitive impairment on regional cerebellar GMV. We used the general linear model (GLM) approach, as implemented in SPM 12, to investigate the effect of group (remitted MDD with cognitive deficits, remitted MDD without cognitive deficits, HC) upon voxel-wise GMV, resulting in voxelwise parameter estimates. Age, gender, years of education, and depression severity (i.e., HAMD score [34]) were 
Table 2 Cognitive performance in patients with remitted major depression (rMDD) with or without persistent cognitive deficits (c.d). Raw values from single neuropsychological tests were $z$-transformed and averaged to form composite scores for four cognitive domains, see the "Material and Methods" section for details. In addition, a general composite score was formed; see the "Material and Methods" section for details. The table shows $z$-values. $s d$, standard deviation

\begin{tabular}{|c|c|c|c|c|c|}
\hline & \multicolumn{2}{|c|}{ rMDD with c.d. $(n=37)$} & \multicolumn{2}{|c|}{ rMDD without c.d. $(n=12)$} & \multirow[b]{2}{*}{$p$ value } \\
\hline & Mean & $\mathrm{sd}$ & Mean & $\mathrm{sd}$ & \\
\hline Attention & -0.24 & 0.54 & 0.46 & 0.52 & $<0.001^{\mathrm{a}}$ \\
\hline Processing speed & -0.25 & 0.94 & 0.38 & 0.64 & $0.036^{\mathrm{a}}$ \\
\hline Learning and memory & -0.12 & 0.73 & 0.07 & 0.82 & $0.445^{\mathrm{a}}$ \\
\hline Executive function & -0.25 & 0.61 & 0.27 & 0.36 & $0.008^{\mathrm{a}}$ \\
\hline General composite score & -0.22 & 0.55 & 0.30 & 0.49 & $0.006^{\mathrm{a}}$ \\
\hline
\end{tabular}

${ }^{\mathrm{a}} t$ test

included as nuisance variables. For completeness, an additional GLM was calculated, where depression severity was not included as a nuisance variable. To investigate betweengroup differences, voxel-wise $t$ tests were calculated. As in previous studies $[35,36]$, given a strong a priori hypothesis, we chose a significance threshold of $p<0.005$ (uncorrected at the voxel level) to assess between-group differences. Based on random field theory [37], an empirically determined extent threshold according to the expected number of voxels per cluster within the respective contrast was applied. Anatomical localizations were determined using a probabilistic MRI atlas of the cerebellum [38], as implemented in the SPM Anatomy Toolbox [39]. For a graphical summary of results, color-coded statistical maps were created, see Fig. 1.

\section{Correlations with Clinical and Neuropsychological Scores}

Within the patient group, explorative correlation analyses were calculated between regional morphometric effects and clinical variables (duration of illness and number of depressive episodes), as well as cognitive parameters (four cognitive domain scores and general cognitive composite score). To this end, the first eigenvariate of the parameter estimates was extracted from cerebellar clusters showing between-group differences. Correlations were calculated using the Prism 6 software (http://www.graphpad.com/scientific-software/prism). A nominal threshold of $p<0.05$ was defined (uncorrected for multiple comparisons).

\section{Results}

Compared with HC, patients with remitted MDD and persistent cognitive deficits (rMDD with c.d.) showed a GMV reduction within left area VIIA, crus II, and vermal area VIIB (see Fig. 1 and Table 3). Patients with remitted MDD and without cognitive deficits (rMDD without c.d.) showed a GMV increase within bilateral area VIIIB compared with HC (see Fig. 1 and Table 3). No significant GMV differences were detected between the two patient groups.

In MDD patients, significant correlations were found between GMV of the cluster in left area VIIA, crus II, and attention functioning $(r=0.50, p<0.001)$, executive performance $(r=0.38, p=0.007)$, and general cognitive functioning, i.e., the cognitive composite score $(r=0.36, p=0.012)$. Additionally, significant correlations were found between GMV of the cluster in vermal area VIIB and attention functioning $(r=0.44, p=0.001)$, executive performance $(r=0.42, p=$ $0.003)$, and general cognitive functioning $(r=0.36, p=0.012)$. There were no significant correlations between GMV and clinical variables, i.e., duration of illness or number of depressive episodes. When correcting the correlation analyses for multiple comparisons using the Bonferroni method $(p<0.0071)$, correlations between GMV in both clusters and attention and executive performance, respectively, remained significant, while correlations between GMV in both clusters and general cognitive functioning showed a trend towards significance.

To test for potential effects of depression severity, we excluded the HAMD score as a nuisance variable in the firstlevel analysis and recalculated all second-level analyses. This approach did not change our main findings, except for the cluster in vermal area VIIB in patients with cognitive deficits (see Supplementary Material, Table 1).

\section{Discussion}

This study investigated cerebellar GMV in patients with remitted MDD with or without cognitive deficits compared with healthy controls. Two main findings emerged: First, patients with remitted MDD and cognitive deficits showed reduced GMV within area VIIA, crus II, and vermal area VIIB, which was associated with attention deficits and impaired executive 


\section{rMDD with c.D. < HC}
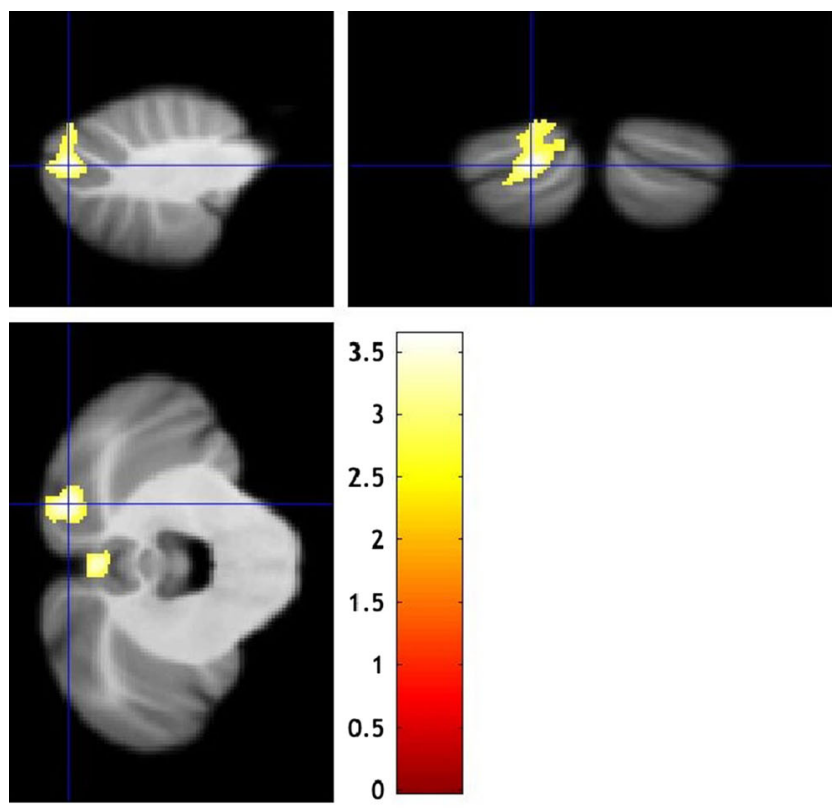

rMDD without c.D. > HC
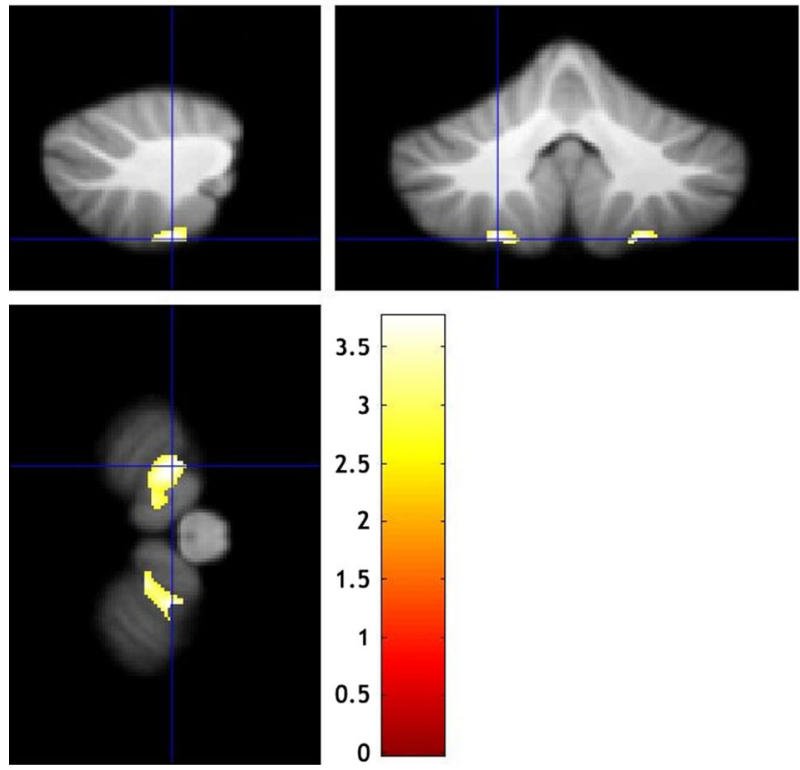

Fig. 1 Cerebellar regions showing aberrant gray matter volume in patients with remitted MDD (rMDD) with or without cognitive deficits (c.d.), each compared with healthy controls (HC). Sagittal, coronal, and horizontal views (clockwise, starting from top left). Results of secondlevel between-group analyses (two-sample $t$ tests; nuisance variables: age, gender, years of education, and depression severity, i.e., HAMDScore), $p<0.005$ uncorrected for height, corrected for spatial extent using an empirically determined threshold of $k>259$ voxels, see the "Material and Methods" section for details. The color bar represents $t$ values

performance. Second, patients with remitted MDD without cognitive deficits showed increased GMV in area VIIIB.
So far, cognitive deficits in depression have mainly been associated with structural and functional changes of cerebral regions [10-13]. In line with our hypothesis, this study revealed abnormal structure of cerebellar area VII in patients with remitted depression, which was linked to cognitive dysfunction. This adds to a recent body of research that consistently demonstrated contributions of area VII to cognitive functioning in MDD [16]. Indeed, cerebellar contributions to cognitive dysfunction in MDD are now better documented than potential cerebellar correlates of abnormal affective and self-referential processing [16].

Our data are in agreement with the notion that recurrence risk in depression may be related to state-independent abnormalities of brain structure and function [40]. Such neural abnormalities may accumulate with increasing number of depression relapses and they may in turn convey an increased risk for relapse [41-43]. Cognitive dysfunction is considered to be one of the clinical mediators of depression relapse [5-7]. In line with this, this study shows cerebellar GMV changes in remitted depression and an association of abnormal cerebellar GMV with cognitive dysfunction. Future longitudinal studies should specifically address whether cerebellar abnormalities persisting into remission may be associated with risk of MDD relapse.

It is important to emphasize that performance deficits that can be assessed in neuropsychological tests represent only one facet of cognitive dysfunction in depression. At the core of cognitive depression theories, cognitive dysfunction is being related to preferential processing of negative emotional information and dysfunctional emotion regulation, e.g. rumination $[44,45]$. Future studies should investigate potential cerebellar contributions to these depression-specific cognitive processes. Schmahmann and colleagues have suggested that cerebellar dysfunction may lead to "cognitive-affective dysmetria", i.e., to specific abnormalities of cognitive and affective processes, in analogy to the well-defined "motor dysmetria" in cerebellar disorders [46]. This is a highly attractive model, but empirical support in patients with MDD is lacking at present. So far, no studies have investigated the mechanisms by which cerebellar abnormalities in MDD may be related to negative cognitive styles or impaired cognitive control. At this point, such cognitive abnormalities have only been associated with dysfunction of the cerebral components of the so-called cognitive control network $[47,48]$. In future studies, potential associations between depressive cognition and abnormal cerebellar function or abnormal cerebro-cerebellar functional connectivity should be of particular interest [22]. Such investigations will help to validate and refine the model of "cognitive-affective dysmetria" as a result of cerebellar dysfunction.

In MDD treatment, non-invasive regional brain stimulation, e.g., transcranial magnetic stimulation (TMS), promises to reduce depressive symptoms by modulating neural activity in brain regions associated with cognitive processes [49]. 
Table 3 Cerebellar regions showing aberrant gray matter volume in patients with remitted major depression (rMDD) with or without cognitive deficits (c.d.), each compared with healthy controls (HC). The table shows $t$ values and stereotaxic coordinates $(x, y, z)$ for peak voxels emerging from second-level between-group comparisons, see the "Material and
Methods" section for details. Peak voxel coordinates were assigned to probabilistic cytoarchitectonic maps, providing the probability and the $95 \%$ confidence range of belonging to a specific cerebellar area. SUIT, Spatially Unbiased Infratentorial Toolbox

\begin{tabular}{|c|c|c|c|c|c|c|}
\hline & \multicolumn{5}{|l|}{ SUIT analysis } & \multirow[b]{2}{*}{ No. of voxels } \\
\hline & Cerebellar region & $x$ & $y$ & $z$ & $t$-value & \\
\hline \multirow[t]{2}{*}{ rMDD with c.d. $<$ HC } & $\begin{array}{l}\text { Left area VIIA, crus II } \\
(69 \%, 12-69 \%) \\
\text { Left area VIIA, crus I } \\
(31 \%, 31-84 \%)\end{array}$ & -17 & -83 & -34 & 3.62 & 1128 \\
\hline & $\begin{array}{l}\text { Vermal area VIIB } \\
(56 \%, 11-56 \%)\end{array}$ & 1 & -74 & -34 & 3.40 & 451 \\
\hline \multirow[t]{2}{*}{ rMDD without c.d. > HC } & $\begin{array}{l}\text { Left area VIIIb } \\
(92 \%, 83-95 \%)\end{array}$ & -21 & -51 & -60 & 3.76 & 444 \\
\hline & $\begin{array}{l}\text { Right area VIIIb } \\
(96 \%, 76-96 \%)\end{array}$ & 22 & -52 & -60 & 3.75 & 282 \\
\hline
\end{tabular}

The cerebellar region printed in italics belongs to the voxel coordinates provided in the row above, i.e. there is a $69 \%$ probability that $\mathrm{x}=-17, \mathrm{y}=-83, \mathrm{z}=-$ 24 belongs to left area VIIA, crus II (top row), and a $31 \%$ probability that $\mathrm{x}=-17, \mathrm{y}=-83, \mathrm{z}=-24$ belongs to left area VIIA, crus I (lower row)

TMS is typically applied over the lateral frontal cortex [50]. In light of our findings, cerebellar area VII should receive further attention as a possible target for TMS. In fact, pilot studies that applied TMS to the cerebellum are available [51-53].

In this study, patients with remitted MDD that showed intact cognitive functioning displayed increased volume of cerebellar area VIIIB compared with controls. Area VIIIB is associated with sensorimotor functions [54]. Psychomotor retardation is a major feature of MDD [55], but its neural correlates have been addressed by only a few studies [56, 57]. We have previously detected abnormal resting state perfusion in area VIIIB in acutely depressed patients [21]. We did not systematically assess psychomotor symptoms in this study (nor in our previous study), so we cannot make inferences about a potential psychomotor impact of abnormal area VIIIB structure (or function) in MDD. Interestingly, in patients with acute major depression, we previously found increased GMV in different cerebellar subregions $[19,20]$. The causes of increased cerebellar GMV in MDD are unknown at this point of research. However, given that cerebellar plasticity is well recognized, it may be speculated that increased cerebellar GMV in depression may reflect compensation efforts, i.e., the so-called cerebellar reserve [58].

We acknowledge potential limitations of our study. Psychopharmacological treatment in patients was heterogeneous. Medication effects on cerebellar structure cannot be excluded. Also, more female than male subjects participated in this study. The disproportion was greatest in the subgroup of MDD patients without cognitive deficits. To correct for possible gender effects, gender was included as a nuisance variable in the statistical analysis of morphometric effects. Neuropsychological evaluation was not performed in healthy controls, preventing cerebellar structure-cognition analyses in those subjects. No patients with treatment-resistant depression were investigated in this study; thus, no claims can be made about abnormal cerebellar structure in such an MDD subgroup. Also, this study did not include patients with geriatric depression, where depressive symptoms and cognitive dysfunction may arise from accelerated brain aging. Thus, age-related cerebellar abnormalities in MDD cannot be addressed. Eventually, due to the cross-sectional study design, no conclusions can be made about the temporal stability of the cerebellar GMV findings.

\section{Conclusion}

Keeping potential shortcomings in mind, this study demonstrated that MDD patients with current depressive symptom remission had abnormal GMV in both hemispheric and vermal portions of cerebellar area VII, which was associated with deficits of attention and executive function. The data support relevant contributions of the cerebellum to the cognitive dimension of MDD. Future studies should address whether abnormal area VII structure is associated with increased risk of depression relapse, whether abnormal area VII structure is linked to abnormal cognitive processes in depression (such as preferential processing of negative information or dysfunctional explicit emotion regulation), and whether abnormal area VII structure is associated with abnormal cerebellar activity or with abnormal cerebro-cerebellar functional connectivity. Future studies will particularly benefit from investigating MDD patients along a spectrum of cognitive functioning, as promoted by the Research Domain Criteria Initiative (RDoC) [27]. In MDD research, there has been an increasing interest in neural abnormalities at the functional network level [59]. In 
this context, computational approaches hold promise for identification of clinically useful biomarkers $[60,61]$. Our results suggest that the cerebellum should be included in future analyses of neural networks in MDD, particularly when the cognitive dimension of depression is investigated. Finally, our results indicate that cerebellar area VII should be evaluated as a target for brain stimulation to treat cognitive deficits related to MDD.

Authors Contributions RCW and DRE designed and supervised the study. MSD, CB, LL, and JK performed the experiments. MSD, MMS, LL, DRE, and RCW analyzed the data. KMK contributed to interpretation of data. MSD and RCW wrote the manuscript. All authors read and approved the final manuscript.

Funding Information Open Access funding enabled and organized by Projekt DEAL. This study was funded by the German Research Foundation (DFG; grant numbers: WO 1883/4-1 to RCW, RO 3418/61 to DRE).

\section{Compliance with Ethical Standards}

The study was approved by the local ethics committee (Heidelberg University) and carried out in accordance with the Helsinki Declaration. Written informed consent was obtained from all participants.

Conflict of Interest DRE has contracts for the development of neuropsychological diagnostic and training tools with Schuhfried GmbH. The other authors declare that they have no conflict of interest.

Open Access This article is licensed under a Creative Commons Attribution 4.0 International License, which permits use, sharing, adaptation, distribution and reproduction in any medium or format, as long as you give appropriate credit to the original author(s) and the source, provide a link to the Creative Commons licence, and indicate if changes were made. The images or other third party material in this article are included in the article's Creative Commons licence, unless indicated otherwise in a credit line to the material. If material is not included in the article's Creative Commons licence and your intended use is not permitted by statutory regulation or exceeds the permitted use, you will need to obtain permission directly from the copyright holder. To view a copy of this licence, visit http://creativecommons.org/licenses/by/4.0/.

\section{References}

1. Rock PL, Roiser JP, Riedel WJ, Blackwell AD. Cognitive impairment in depression: a systematic review and meta-analysis. Psychol Med. 2014;44:2029-40. https://doi.org/10.1017/ s0033291713002535.

2. McIntyre RS, Cha DS, Soczynska JK, Woldeyohannes HO, Gallaugher LA, Kudlow P, et al. Cognitive deficits and functional outcomes in major depressive disorder: determinants, substrates, and treatment interventions. Depress Anxiety. 2013;30:515-27. https://doi.org/10.1002/da.22063.

3. Trivedi MH, Greer TL. Cognitive dysfunction in unipolar depression: implications for treatment. J Affect Disord. 2014;152-154:19 27. https://doi.org/10.1016/j.jad.2013.09.012.

4. Hasselbalch BJ, Knorr U, Kessing LV. Cognitive impairment in the remitted state of unipolar depressive disorder: a systematic review. J
Affect Disord. 2011;134:20-31. https://doi.org/10.1016/j.jad.2010. 11.011.

5. Alexopoulos GS, Meyers BS, Young RC, Kalayam B, Kakuma T, Gabrielle M, et al. Executive dysfunction and long-term outcomes of geriatric depression. Arch Gen Psychiatry. 2000;57:285-90. https://doi.org/10.1001/archpsyc.57.3.285.

6. Lee RS, Hermens DF, Porter MA, Redoblado-Hodge MA. A metaanalysis of cognitive deficits in first-episode major depressive disorder. J Affect Disord. 2012;140:113-24. https://doi.org/10.1016/j. jad.2011.10.023.

7. Majer M, Ising M, Kunzel H, Binder EB, Holsboer F, Modell S, et al. Impaired divided attention predicts delayed response and risk to relapse in subjects with depressive disorders. Psychol Med. 2004;34:1453-63. https://doi.org/10.1017/s0033291704002697.

8. Bortolato B, Miskowiak KW, Kohler CA, Maes M, Fernandes BS, Berk M, et al. Cognitive remission: a novel objective for the treatment of major depression? BMC Med. 2016;14:9. https://doi.org/ 10.1186/s12916-016-0560-3.

9. Listunova L, Roth C, Bartolovic M, Kienzle J, Bach C, Weisbrod $\mathrm{M}$, et al. Cognitive impairment along the course of depression: nonpharmacological treatment options. Psychopathology. 2018;51: 295-305. https://doi.org/10.1159/000492620.

10. Diener C, Kuehner C, Brusniak W, Ubl B, Wessa M, Flor H. A meta-analysis of neurofunctional imaging studies of emotion and cognition in major depression. Neuroimage. 2012;61:677-85. https://doi.org/10.1016/j.neuroimage.2012.04.005.

11. Pizzagalli DA. Frontocingulate dysfunction in depression: toward biomarkers of treatment response. Neuropsychopharmacology. 2011;36:183-206. https://doi.org/10.1038/npp.2010.166.

12. Vasic N, Walter H, Hose A, Wolf RC. Gray matter reduction associated with psychopathology and cognitive dysfunction in unipolar depression: a voxel-based morphometry study. J Affect Disord. 2008;109:107-16. https://doi.org/10.1016/j.jad.2007.11.011.

13. Vasic N, Walter H, Sambataro F, Wolf RC. Aberrant functional connectivity of dorsolateral prefrontal and cingulate networks in patients with major depression during working memory processing. Psychol Med. 2009;39:977-87. https://doi.org/10.1017/ s0033291708004443.

14. Alalade E, Denny K, Potter G, Steffens D, Wang L. Altered cerebellar-cerebral functional connectivity in geriatric depression. PLoS One. 2011;6:e20035. https://doi.org/10.1371/journal.pone. 0020035.

15. Strick PL, Dum RP, Fiez JA. Cerebellum and nonmotor function. Annu Rev Neurosci. 2009;32:413-34. https://doi.org/10.1146/ annurev.neuro.31.060407.125606.

16. Depping MS, Schmitgen MM, Kubera KM, Wolf RC. Cerebellar contributions to major depression. Frontiers in psychiatry. 2018;9: 634. https://doi.org/10.3389/fpsyt.2018.00634.

17. Kuhn S, Romanowski A, Schilling C, Banaschewski T, Barbot A, Barker GJ, et al. Manual dexterity correlating with right lobule VI volume in right-handed 14-year-olds. Neuroimage. 2012;59:161521. https://doi.org/10.1016/j.neuroimage.2011.08.100.

18. Kuhn S, Romanowski A, Schubert F, Gallinat J. Reduction of cerebellar grey matter in Crus I and II in schizophrenia. Brain Struct Funct. 2012;217:523-9. https://doi.org/10.1007/s00429-011-03652.

19. Depping MS, Nolte HM, Hirjak D, Palm E, Hofer S, Stieltjes B, et al. Cerebellar volume change in response to electroconvulsive therapy in patients with major depression. Prog NeuroPsychopharmacol Biol Psychiatry. 2017;73:31-5. https://doi.org/ 10.1016/j.pnpbp.2016.09.007.

20. Depping MS, Wolf ND, Vasic N, Sambataro F, Hirjak D, Thomann PA, et al. Abnormal cerebellar volume in acute and remitted major depression. Prog Neuro-Psychopharmacol Biol Psychiatry. 2016;71:97-102. https://doi.org/10.1016/j.pnpbp.2016.06.005. 
21. Depping MS, Wolf ND, Vasic N, Sosic-Vasic Z, Schmitgen MM, Sambataro F, et al. Aberrant resting-state cerebellar blood flow in major depression. J Affect Disord. 2018;226:227-31. https://doi. org/10.1016/j.jad.2017.09.028.

22. Guo W, Liu F, Liu J, Yu L, Zhang Z, Zhang J, et al. Is there a cerebellar compensatory effort in first-episode, treatment-naive major depressive disorder at rest? Prog Neuro-Psychopharmacol Biol Psychiatry. 2013;46:13-8. https://doi.org/10.1016/j.pnpbp.2013. 06.009 .

23. Guo W, Liu F, Liu J, Yu M, Zhang Z, Liu G, et al. Increased cerebellar-default-mode-network connectivity in drug-naive major depressive disorder at rest. Medicine. 2015;94:e560. https://doi.org/ 10.1097/md.0000000000000560.

24. Guo W, Liu F, Xue Z, Gao K, Liu Z, Xiao C, et al. Abnormal resting-state cerebellar-cerebral functional connectivity in treatment-resistant depression and treatment sensitive depression. Prog Neuro-Psychopharmacol Biol Psychiatry. 2013;44:51-7. https://doi.org/10.1016/j.pnpbp.2013.01.010.

25. Liu L, Zeng LL, Li Y, Ma Q, Li B, Shen H, et al. Altered cerebellar functional connectivity with intrinsic connectivity networks in adults with major depressive disorder. PLoS One. 2012;7:e39516. https://doi.org/10.1371/journal.pone.0039516.

26. Ma Q, Zeng LL, Shen H, Liu L, Hu D. Altered cerebellar-cerebral resting-state functional connectivity reliably identifies major depressive disorder. Brain Res. 2013;1495:86-94. https://doi.org/10. 1016/j.brainres.2012.12.002.

27. Insel T, Cuthbert B, Garvey M, Heinssen R, Pine DS, Quinn K, et al. Research domain criteria (RDoC): toward a new classification framework for research on mental disorders. Am J Psychiatry. 2010;167:748-51. https://doi.org/10.1176/appi.ajp.2010. 09091379 .

28. Listunova L, Bartolovic M, Kienzle J, Jaehn A, Grutzner TM, Wolf $\mathrm{RC}$, et al. Predictors of cognitive remediation therapy improvement in (partially) remitted unipolar depression. J Affect Disord. 2020;264:40-9. https://doi.org/10.1016/j.jad.2019.12.006.

29. Lehrl S, Triebig G, Fischer B. Multiple choice vocabulary test MWT as a valid and short test to estimate premorbid intelligence. Acta Neurol Scand. 1995;91:335-45. https://doi.org/10.1111/j. 1600-0404.1995.tb07018.x.

30. Ashburner J, Friston KJ. Unified segmentation. Neuroimage. 2005;26:839-51. https://doi.org/10.1016/j.neuroimage.2005.02. 018.

31. Ashburner J. A fast diffeomorphic image registration algorithm. Neuroimage. 2007;38:95-113. https://doi.org/10.1016/j. neuroimage.2007.07.007.

32. Radua J, Canales-Rodriguez EJ, Pomarol-Clotet E, Salvador R. Validity of modulation and optimal settings for advanced voxelbased morphometry. Neuroimage. 2014;86:81-90. https://doi.org/ 10.1016/j.neuroimage.2013.07.084.

33. Jones DK, Symms MR, Cercignani M, Howard RJ. The effect of filter size on VBM analyses of DT-MRI data. Neuroimage. 2005;26:546-54. https://doi.org/10.1016/j.neuroimage.2005.02. 013.

34. Hamilton M. A rating scale for depression. J Neurol Neurosurg Psychiatry. 1960;23:56-62.

35. Cierpka M, Wolf ND, Kubera KM, Schmitgen MM, Vasic N, Frasch K, et al. Cerebellar contributions to persistent auditory verbal hallucinations in patients with schizophrenia. Cerebellum. 2017;16:964-72. https://doi.org/10.1007/s12311-017-0874-5.

36. Kramer J, Huber M, Mundinger C, Schmitgen MM, Pycha R, Kirchler E, et al. Abnormal cerebellar volume in somatic vs nonsomatic delusional disorders. Cerebellum Ataxias. 2020;7:2. https://doi.org/10.1186/s40673-020-0111-8.

37. Hayasaka S, Nichols TE. Validating cluster size inference: random field and permutation methods. Neuroimage. 2003;20:2343-56. https://doi.org/10.1016/j.neuroimage.2003.08.003.
38. Diedrichsen J. A spatially unbiased atlas template of the human cerebellum. Neuroimage. 2006;33:127-38. https://doi.org/10. 1016/j.neuroimage.2006.05.056.

39. Eickhoff SB, Stephan KE, Mohlberg H, Grefkes C, Fink GR, Amunts K, et al. A new SPM toolbox for combining probabilistic cytoarchitectonic maps and functional imaging data. neuroimage. 2005;25:1325-35. https://doi.org/10.1016/j.neuroimage.2004.12. 034.

40. Post RM, Weiss SR. Sensitization and kindling phenomena in mood, anxiety, and obsessive-compulsive disorders: the role of serotonergic mechanisms in illness progression. Biol Psychiatry. 1998;44:193-206. https://doi.org/10.1016/s0006-3223(98)001449.

41. Davey CG, Whittle S, Harrison BJ, Simmons JG, Byrne ML, Schwartz OS, et al. Functional brain-imaging correlates of negative affectivity and the onset of first-episode depression. Psychol Med. 2015;45:1001-9. https://doi.org/10.1017/s0033291714002001.

42. Jacobs RH, Barba A, Gowins JR, Klumpp H, Jenkins LM, Mickey BJ, et al. Decoupling of the amygdala to other salience network regions in adolescent-onset recurrent major depressive disorder. Psychol Med. 2016;46:1055-67. https://doi.org/10.1017/ s0033291715002615.

43. Liu CH, Tang LR, Gao Y, Zhang GZ, Li B, Li M, et al. Restingstate mapping of neural signatures of vulnerability to depression relapse. J Affect Disord. 2019;250:371-9. https://doi.org/10.1016/ j.jad.2019.03.022.

44. Gotlib IH, Joormann J. Cognition and depression: current status and future directions. Annu Rev Clin Psychol. 2010;6:285-312. https:// doi.org/10.1146/annurev.clinpsy.121208.131305.

45. Joormann J, Quinn ME. Cognitive processes and emotion regulation in depression. Depress Anxiety. 2014;31:308-15. https://doi. org/10.1002/da.22264.

46. Schmahmann JD, Weilburg JB, Sherman JC. The neuropsychiatry of the cerebellum - insights from the clinic. Cerebellum. 2007;6: 254-67. https://doi.org/10.1080/14734220701490995.

47. Jacobs RH, Jenkins LM, Gabriel LB, Barba A, Ryan KA, Weisenbach SL, et al. Increased coupling of intrinsic networks in remitted depressed youth predicts rumination and cognitive control. PLoS One. 2014;9:e104366. https://doi.org/10.1371/journal. pone.0104366.

48. Stange JP, Bessette KL, Jenkins LM, Peters AT, Feldhaus C, Crane NA, et al. Attenuated intrinsic connectivity within cognitive control network among individuals with remitted depression: temporal stability and association with negative cognitive styles. Hum Brain Mapp. 2017;38:2939-54. https://doi.org/10.1002/hbm.23564.

49. Tik M, Hoffmann A, Sladky R, Tomova L, Hummer A, Navarro de Lara L, et al. Towards understanding rTMS mechanism of action: stimulation of the DLPFC causes network-specific increase in functional connectivity. Neuroimage. 2017;162:289-96. https://doi.org/ 10.1016/j.neuroimage.2017.09.022.

50. Perera T, George MS, Grammer G, Janicak PG, Pascual-Leone A, Wirecki TS. The clinical TMS society consensus review and treatment recommendations for TMS therapy for major depressive disorder. Brain Stimul. 2016;9:336-46. https://doi.org/10.1016/j.brs. 2016.03.010.

51. De Vidovich GZ, Muffatti R, Monaco J, Caramia N, Broglia D, Caverzasi E, et al. Repetitive TMS on left cerebellum affects impulsivity in borderline personality disorder: a pilot study. Front Hum Neurosci. 2016;10:582. https://doi.org/10.3389/fnhum.2016. 00582.

52. Rastogi A, Cash R, Dunlop K, Vesia M, Kucyi A, Ghahremani A, et al. Modulation of cognitive cerebello-cerebral functional connectivity by lateral cerebellar continuous theta burst stimulation. Neuroimage. 2017;158:48-57. https://doi.org/10.1016/j. neuroimage.2017.06.048. 
53. van Dun K, Bodranghien F, Manto M, Marien P. Targeting the cerebellum by noninvasive neurostimulation: a review. Cerebellum. 2017;16:695-741. https://doi.org/10.1007/s12311016-0840-7.

54. Stoodley CJ, Schmahmann JD. Functional topography in the human cerebellum: a meta-analysis of neuroimaging studies. Neuroimage. 2009;44:489-501. https://doi.org/10.1016/j. neuroimage.2008.08.039.

55. Buyukdura JS, McClintock SM, Croarkin PE. Psychomotor retardation in depression: biological underpinnings, measurement, and treatment. Prog Neuro-Psychopharmacol Biol Psychiatry. 2011;35: 395-409. https://doi.org/10.1016/j.pnpbp.2010.10.019.

56. Liberg B, Rahm C. The functional anatomy of psychomotor disturbances in major depressive disorder. Front Psychiatry. 2015;6:34. https://doi.org/10.3389/fpsyt.2015.00034.

57. Yin $\mathrm{Y}$, Wang M, Wang Z, Xie C, Zhang H, Zhang $\mathrm{H}$, et al. Decreased cerebral blood flow in the primary motor cortex in major depressive disorder with psychomotor retardation. Prog NeuroPsychopharmacol Biol Psychiatry. 2018;81:438-44. https://doi. org/10.1016/j.pnpbp.2017.08.013.
58. Mitoma H, Buffo A, Gelfo F, Guell X, Fuca E, Kakei S, et al. Consensus Paper. Cerebellar reserve: from cerebellar physiology to cerebellar disorders. Cerebellum. 2020;19:131-53. https://doi. org/10.1007/s12311-019-01091-9.

59. Drysdale AT, Grosenick L, Downar J, Dunlop K, Mansouri F, Meng Y, et al. Resting-state connectivity biomarkers define neurophysiological subtypes of depression. Nat Med. 2017;23:28-38. https://doi.org/10.1038/nm.4246.

60. Crane NA, Jenkins LM, Bhaumik R, Dion C, Gowins JR, Mickey $\mathrm{BJ}$, et al. Multidimensional prediction of treatment response to antidepressants with cognitive control and functional MRI. Brain. 2017;140:472-86. https://doi.org/10.1093/brain/aww326.

61. van Waarde JA, Scholte HS, van Oudheusden LJ, Verwey B, Denys D, van Wingen GA. A functional MRI marker may predict the outcome of electroconvulsive therapy in severe and treatmentresistant depression. Mol Psychiatry. 2015;20:609-14. https://doi. org/10.1038/mp.2014.78.

Publisher's Note Springer Nature remains neutral with regard to jurisdictional claims in published maps and institutional affiliations. 